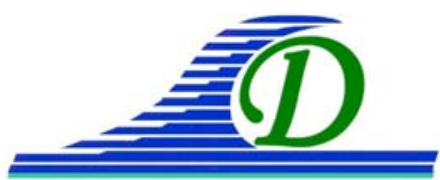
XIII ${ }^{\text {èmes }}$ Journées Nationales Génie Côtier - Génie Civil Dunkerque, 2-4 juillet 2014

DOI:10.5150/jngcgc.2014.082 (c) Editions Paralia CFL

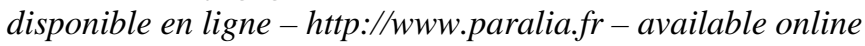

\title{
Projet EMACOP : évaluation du potentiel houlomoteur de 22 sites français en Manche et Atlantique
}

\section{Bertrand MICHARD ${ }^{1}$, Emmanuel COSQUER ${ }^{1}$, Gaëtan DUFOUR ${ }^{2}$}

1. CEREMA / DTecEMF, Technopôle Brest-Iroise BP 5, 29280 Plouzané, France. bertrand.michard@cerema.fr ; emmanuel.cosquer@cerema.fr

2. International Marine and Dredging Consultants (IMDC), 4 Coveliersstraat B-2600 Berchem (Antwerp), Belgique.

gaetan.dufour@imdc.be

\section{Résumé :}

Dans le cadre du projet national de recherche EMACOP (Energies MArines COtières et Portuaires), une méthode de calcul analytique a été employée pour propager la houle du large à la côte (GODA, 2000), à partir de l'analyse statistique de données de houle de l'atlas numérique ANEMOC (BENOIT et al., 2008). Elle a permis de caractériser le potentiel houlomoteur de 22 sites côtiers et portuaires des façades maritimes de la Manche et de l'Atlantique.

La classification est établie à partir de l'analyse des résultats d'étude présentés dans le tableau récapitulatif et les fiches de sites. L’exemple de Saint-Guénolé est ici repris. Rappelons que les critères de bathymétrie, de puissances de houle annuelle et hivernale et de longueur de digue pouvant être équipée d'un système houlomoteur, ont été particulièrement examinés.

L'évaluation a permis de retenir 9 sites de fort potentiel sur les façades Manche et Atlantique, avec des niveaux de puissance de houle les plus forts de 20 à $25 \mathrm{~kW} / \mathrm{m}$ sur les sites de la pointe Bretagne et du Pays Basque.

Mots-clés : EMACOP, Énergies marines, Potentiel, Houlomoteur, Sites côtiers et portuaires, ANEMOC.

\section{Introduction}

Le projet national de recherche EMACOP (Energies MArines COtières et Portuaires) a pour objectif d'étudier et de promouvoir le développement de systèmes de récupération d'énergie marine adaptés aux structures portuaires et côtières (http://www.emacop.fr/).

Un des objectifs du thème "systèmes houlomoteurs sur ouvrages anciens" est d'évaluer le potentiel houlomoteur de 22 sites français identifiés afin de sélectionner les sites les plus pertinents pouvant être équipés de systèmes récupérateurs d’énergie des vagues.

La sélection et la caractérisation des sites ont été effectuées notamment à partir des critères de bathymétrie, de niveau d'eau, de climats de houle, de niveau de puissance de houle (en $\mathrm{kW} / \mathrm{m}$ ) et de longueur de digue pouvant être équipée. 


\section{Définitions}

\subsection{Niveau de puissance de houle}

Un état de mer réel est constitué de vagues pouvant varier considérablement en hauteur, période et direction de provenance. La répartition de l'énergie de l'état de mer sur les fréquences et sur les directions peut être décrite par la variable de l'équation (1) appelée spectre directionnel d'énergie $E(f, \theta)$, qui se décompose en un spectre de fréquence $S(f)$ et une fonction de répartition angulaire $D(f, \theta)$ (EQUIMAR, 2011) :

$$
E(f, \theta)=S(f) . D(f, \theta)
$$

Le niveau de puissance de houle est la mesure de puissance disponible par unité de longueur d'onde dans une direction qui s'exprime en $\mathrm{kW} / \mathrm{m}$ par l'équation (2) :

$$
P_{w}=\rho \cdot g \int S(f) \cdot c_{g} d f
$$

avec $\rho$ la masse volumique de l'eau et $c_{g}$ la vitesse de groupe définie pour la houle régulière.

En eau profonde, i.e. à une profondeur d'eau supérieure à la moitié de la longueur d'onde, le niveau de puissance de houle $P_{w}$ peut être calculé directement par l'équation (3) avec $H_{m 0}$, la hauteur significative spectrale et $T_{e}$ la période énergétique associée :

$$
P_{w}=\frac{\rho \cdot g^{2}}{64 \pi} H_{m 0}^{2} \cdot T_{e}
$$

\subsection{Climats de houle et propagation de la houle}

L'estimation des climats de houle des sites est réalisée à partir de l'analyse statistique de données de houle de l'atlas numérique ANEMOC développé par le CETMEF et EDF/LNHE (BENOIT et al., 2008), et de mesures de bouées directionnelles du réseau CANDHIS, à laquelle ont été associés des calculs analytiques de propagation de la houle du large vers les sites (GODA, 2000). Les houles subissent des déformations dues aux phénomènes de réfraction par les fonds, de shoaling, de déferlement, de réflexion et de diffraction. L'intensité de l'énergie des vagues peut varier considérablement en fonction des configurations bathymétriques.

\section{Méthodologie d'étude}

\subsection{Présentation des sites}

L'identification de sites côtiers ou portuaires des façades maritimes de la Manche et de l'Atlantique a permis de sélectionner 22 sites d'études présentés en figure 1. 


\section{XIII ${ }^{\text {èmes }}$ Journées Nationales Génie Côtier - Génie Civil \\ Dunkerque, 2-4 juillet 2014}
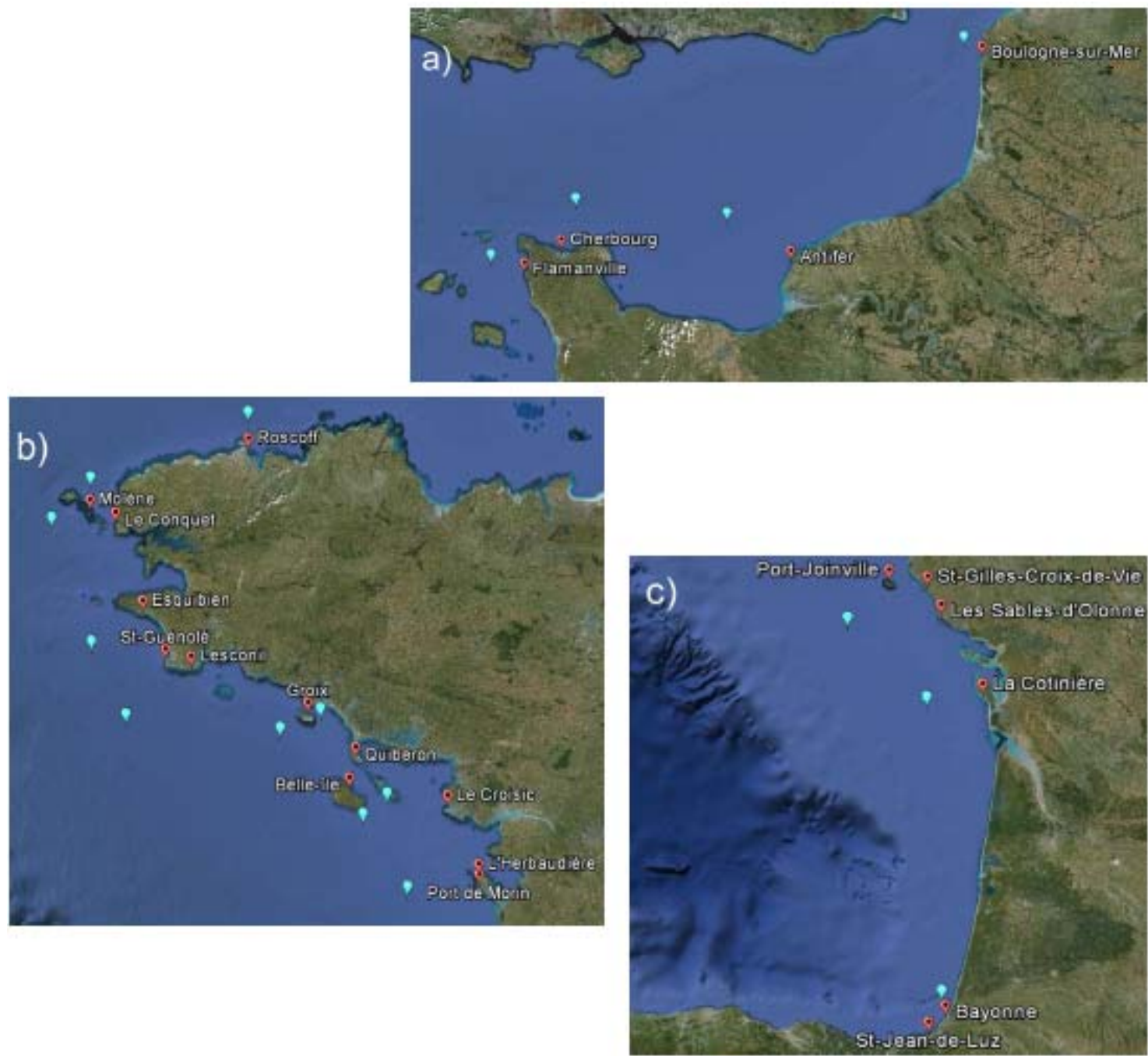

Figure 1. Localisation des sites et des points au large de l'atlas numérique de houle ANEMOC : a) Manche, b) Bretagne et côte Ouest c) côte Sud-Ouest de la France

\subsection{Estimation des climats de houle :}

a) Climats de houle au large

Les données de houle, assimilées à des points de l'atlas numérique ANEMOC, sont élaborées à partir de simulations rétrospectives sur la période de 1979 à 2002 et ont été sélectionnées à des profondeurs de plus de 50 mètres pour fournir les climats de houle au large. Le traitement informatique des fichiers de plus de 200000 lignes correspondant aux valeurs horaires d'états de mer des points au large de l'atlas ANEMOC et de calculer, à partir de la formule (3), le niveau de puissance de houle au large $\mathrm{P}_{\mathrm{w}}$ (MATTAROLO et al., 2009).

La variabilité saisonnière des climats sur les côtes françaises a également conduit à réaliser l'analyse des données suivant les climats de houle annuel, hivernal (d'octobre à mars) et estival (d'avril à septembre) afin de bien caractériser le potentiel houlomoteur. 
b) Climats de houle sur les sites

Les calculs de propagation de la houle du large vers les sites ont été élaborés à partir des formules analytiques (GODA, 2000) qui prennent en compte la réfraction, le shoaling et le déferlement pour les climats de houle annuel, hivernal et estival. Les résultats de calcul fournissent les paramètres de houle à la côte qui permettent de déterminer le niveau de puissance de houle à la côte $P_{c}$ :

$$
P_{c}=\frac{\rho \cdot g}{8} H_{c}^{2} \cdot c_{g}
$$

\section{3 Évaluation du potentiel houlomoteur des sites :}

a) Première phase d'évaluation

L'évaluation a été effectuée à partir des critères de caractérisation de site (cf. tableau 1), et en particulier des critères de bathymétrie (en m CM), de niveaux de puissance de houle annuelle et hivernale (en $\mathrm{kW} / \mathrm{m}$ ) et de longueur de digue pouvant être équipée (en $\mathrm{m})$. Elle a permis de retenir 14 sites susceptibles de présenter un niveau de potentiel intéressant et d'écarter 8 sites présentant de faibles niveaux de puissance de houle, inférieurs à 2,5 kW/m pour 6 d'entre eux ; il s'agit des sites de Molène, Groix, Belle-Ile, Le Croisic, Port de Morin, L’Herbaudière, Port-Joinville et La Cotinière.

b) Deuxième phase d'évaluation

Cette phase a été réalisée à partir de l'analyse détaillée des résultats statistiques de potentiel des 14 sites sélectionnés présentés sous la forme (cf. figures 2,3 et 4) :

- de distributions d'occurrence (en heures par an) pour les états de mer du site définis par la hauteur de houle $H_{c}$ devant le site et la période énergétique $T_{e}$;

- de distributions d'énergie de houle (en $\mathrm{MWh} / \mathrm{m}$ ) par an ;

- de distributions cumulatives des occurrences et de l'énergie annuelle de $P_{c}, H_{c}, T_{e}$, et de direction de provenance ( ${ }^{\circ} /$ Nord), (LENEE-BLUHM et al., 2011) ;

- et d'évolution mensuelle du niveau de puissance de houle pour le point au large de l'atlas ANEMOC et le site d'étude.

Cette phase a permis de sélectionner 9 sites d'étude présentant de forts niveaux de potentiel houlomoteur mais qui restent hétérogènes, car dépendant fortement de la localisation et de la longueur de digue pouvant être équipée.

Ainsi, les estimations de niveaux de puissance de houle annuelle varient de 4,4 kW/m à Cherbourg jusqu'à 24,3 kW/m à Bayonne et les longueurs de digues utiles varient de $140 \mathrm{~m}$ au Conquet, $500 \mathrm{~m}$ à Saint-Jean-de-Luz et jusqu'à $4000 \mathrm{~m}$ à Cherbourg.

Les sites de Boulogne-sur-Mer, Roscoff, Lesconil, Quiberon et Les Sables d'Olonne, qui présentent un niveau de potentiel houlomoteur moyen, ont été écartés.

\section{Exemple de résultats présentés pour le site de Saint-Guénolé (Finistère)}

La partie de digue utile retenue correspond à une section de $250 \mathrm{~m}$ de longueur, avec une cote, en pied de digue, de 2 m CM. 


\section{XIII ${ }^{\text {èmes }}$ Journées Nationales Génie Côtier - Génie Civil \\ Dunkerque, 2-4 juillet 2014}

Tableau 1. Résultats sur les sites.

\begin{tabular}{|c|c|c|c|c|c|c|c|c|}
\hline Site & $\begin{array}{r}\text { Bathymé- } \\
\text { trie } \\
\text { (m CaM) }\end{array}$ & 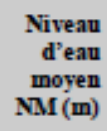 & $\begin{array}{r}\text { Puissance } \\
\text { de houle } \\
\text { annuelle } \\
\text { Pc (lWW/m) }\end{array}$ & $\begin{array}{r}\text { Puissance } \\
\text { de houle } \\
\text { hivernale } \\
(\mathrm{kW} / \mathrm{m})\end{array}$ & $\begin{array}{r}\text { Puissance } \\
\text { de houle } \\
\text { estivale } \\
(\mathrm{kW} / \mathrm{m})\end{array}$ & $\begin{array}{r}\text { Hauteur } \\
\text { de houle } \\
\text { annuelle } \\
\text { Hc (m) }\end{array}$ & $\begin{array}{r}\text { Direction de } \\
\text { provenance } \\
\text { de la houle } \\
(\% / \text { Nord })\end{array}$ & $\begin{array}{l}\text { Longueur de } \\
\text { digue pouvant } \\
\text { être équipée } \\
\text { (m) }\end{array}$ \\
\hline Boul ogne-swr-Mer & 3 & 4,9 & 2,8 & 4,2 & 1,3 & 0,7 & 260 & 1600 \\
\hline Antifer & 16 & 4,87 & 5,1 & 8,2 & 2,2 & 0,9 & 280 & 1900 \\
\hline Cherbourg & 10 & 3,81 & 4,4 & 6,3 & 2,5 & 1 & $300 ; 60$ & 4000 \\
\hline Flamanville & 3 & 5,44 & 13,6 & 22,2 & 5,4 & 1,4 & 270 & 700 \\
\hline Roscoff & 2 & 5,25 & 7,8 & 10,8 & 4,9 & 1,2 & $0 ; 40$ & 300 \\
\hline Molène & 0 & 4,34 & 7,9 & 11,6 & 4,8 & 1,3 & 345 & 60 \\
\hline Le Conquet & 1 & 3,98 & 21,1 & 30,2 & 11,8 & 2,1 & 250 & 140 \\
\hline Esquibien & 2 & 3,07 & 6,9 & 8,4 & 3,4 & 1,3 & 140 & 340 \\
\hline Saint-Guénolé & 2 & 3,01 & 21,1 & 30,2 & 12,6 & 2,1 & 285 & 250 \\
\hline Lescomil & 0 & 3,05 & 8,1 & 9,7 & 5,2 & 1,5 & 190 & 200 \\
\hline Groix & 1 & 3,11 & $\mathrm{x}$ & $\mathrm{x}$ & $\mathrm{x}$ & $\mathrm{x}$ & $\mathrm{x}$ & 200 \\
\hline Quiberon & 0 & 3,13 & 5,8 & 8,2 & 3,2 & 1,1 & 210 & 350 \\
\hline Belle-Ile & 2 & 3,07 & 0,9 & 1 & 0,7 & 0,5 & $25 ; 100$ & 200 \\
\hline Le Croisic & -2 & 3,3 & 1,6 & 1,9 & 1,4 & 0,8 & 315 & 450 \\
\hline Port de Morin & -2 & 3,36 & 2,1 & 2,4 & 1,8 & 0,9 & 235 & 400 \\
\hline L'Herbaudière & -2 & 3,36 & 1,8 & 2,1 & 1,6 & 0,9 & 325 & 100 \\
\hline Port-Joinville & 0 & 3,09 & 1,8 & 2,3 & 1,3 & 0,7 & 10 & 100 \\
\hline St-Gilles-Croix-de-Vie & 1 & 3,17 & 12,2 & 17,9 & 6,6 & 1,6 & 240 & 250 \\
\hline Les Sables d'Olonne & 1 & 3,2 & 10,3 & 13,4 & 5,1 & 1,4 & 210 & 100 \\
\hline La Cotinière & -2 & 3,6 & 2,5 & 3,1 & 1,9 & 1 & 225 & 150 \\
\hline Bayoune & 7 & 2,5 & 24,3 & 37,8 & 11,1 & 1,8 & 300 & 500 \\
\hline Saint-Jean-de-Luz & 10 & 2,48 & 21,8 & 33,8 & 10,1 & 1,6 & 315 & 500 \\
\hline
\end{tabular}

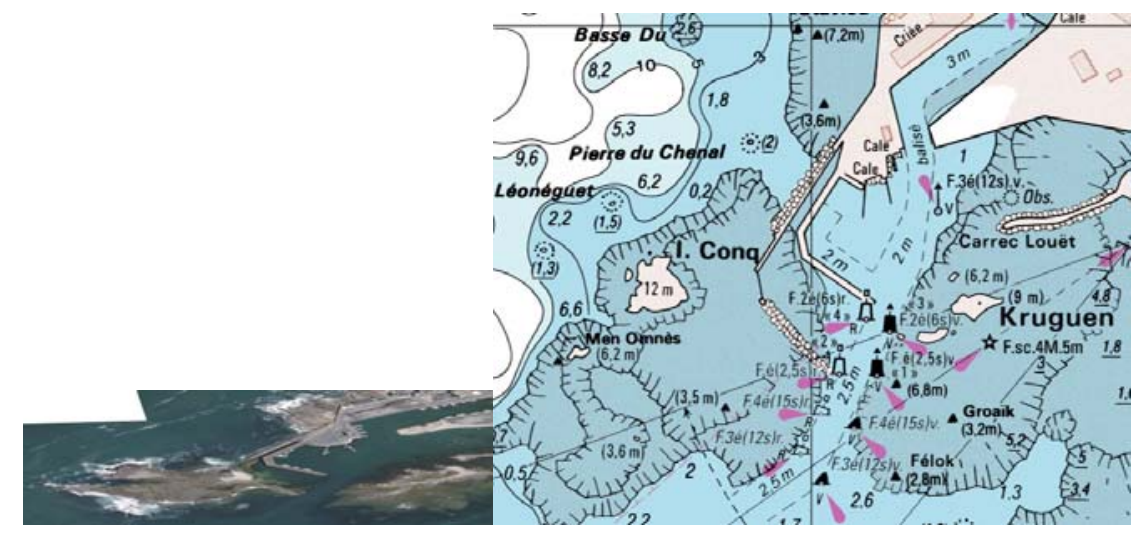

Figure 2. Photographie aérienne du site de Saint-Guénolé (source Géoportail IGN) et extrait de la carte marine $n^{\circ} 6645$ Pointe de Penmarc'h (source SHOM).

Les houles sont très énergétiques le long de la digue $(21,1 \mathrm{~kW} / \mathrm{m}$ en moyenne annuelle et $30,2 \mathrm{~kW} / \mathrm{m}$ en hiver) et arrivent avec une obliquité moyenne sur l'ouvrage de $15^{\circ}$. 


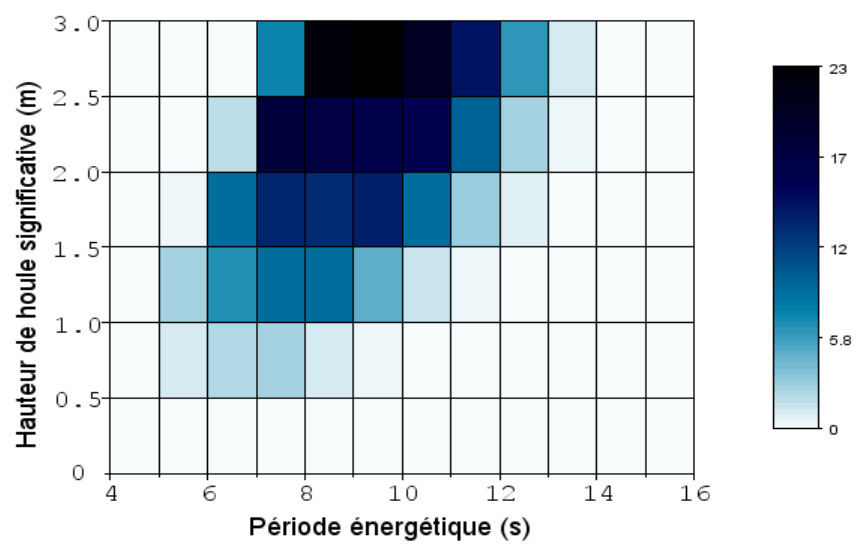

Figure 3. Distribution d'énergie de houle $(M W h / m)$ par an pour les états de mer du site définis par la hauteur de houle devant le site $H_{c}(m)$ et la période énergétique $T_{e}(\mathrm{~s})$.
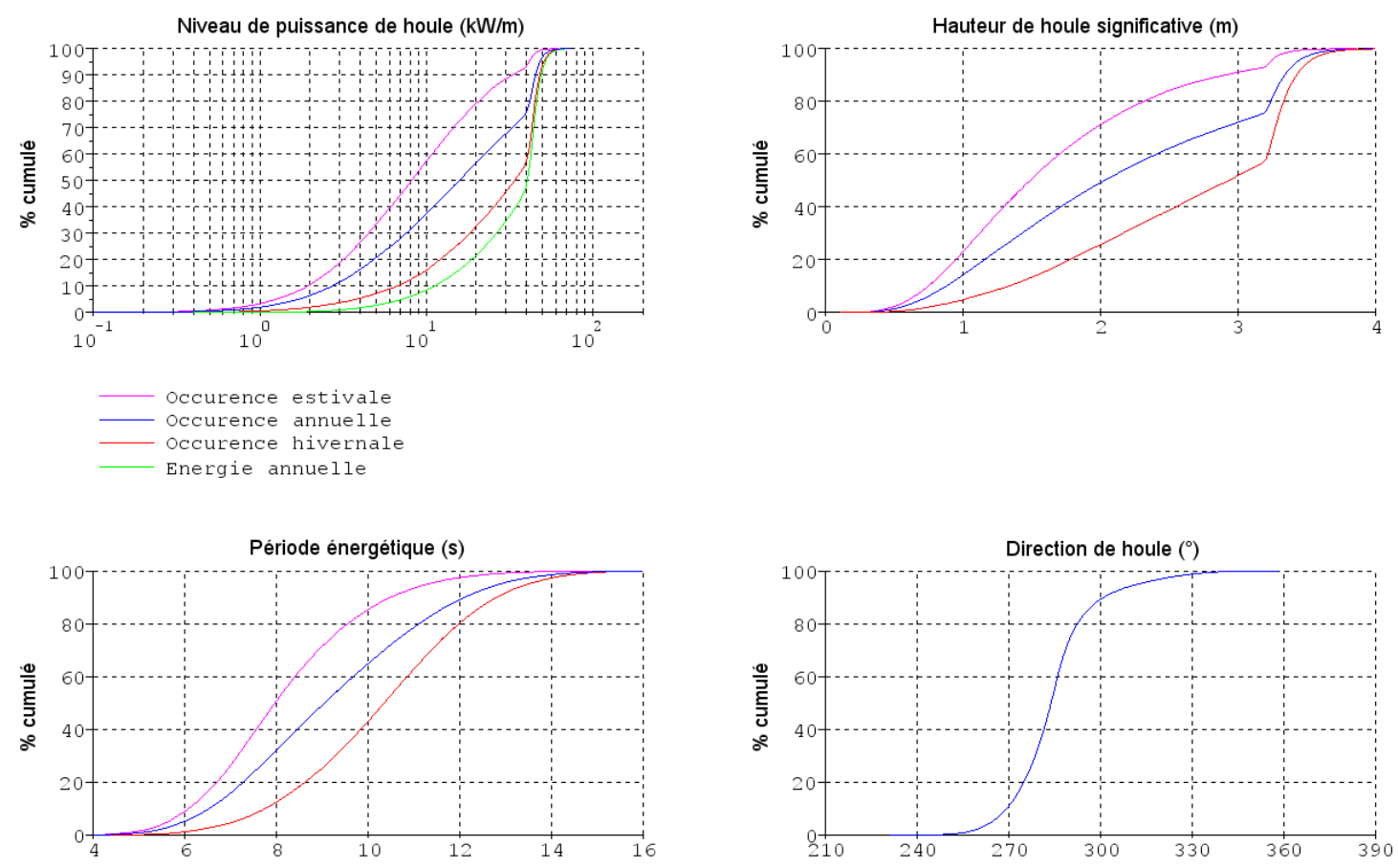

Figure 4. Distributions cumulatives des occurrences (annuelle, hivernale et estivale) et de l'énergie (annuelle) du niveau de puissance de houle $P_{c}(\mathrm{~kW} / \mathrm{m})$, de la hauteur de houle $H_{c}(m)$, de la période énergétique $T_{e}(\mathrm{~s})$ et de la direction de la houle $\left(^{\circ}\right)$.

Le niveau de puissance de houle dépassant le niveau moyen de puissance annuelle (21,1 $\mathrm{kW} / \mathrm{m}$ ) représente $40 \%$ du temps annuel, soit 146 jours par an et compte pour $75 \%$ de l'énergie annuelle. En période hivernale, le niveau de puissance est supérieur à 21,1 $\mathrm{kW} / \mathrm{m}$ pendant $62 \%$ du temps, soit 112 jours. 


\section{XIII ${ }^{\text {èmes }}$ Journées Nationales Génie Côtier - Génie Civil \\ Dunkerque, 2-4 juillet 2014}

\section{Classification des sites}

La classification est établie à partir de l'analyse des résultats présentés dans le tableau récapitulatif (cf. tableau 1) et les fiches de sites détaillées. L'évaluation a permis de sélectionner 9 sites d'études de fort potentiel, répartis sur les façades maritimes de la Manche pour 3 d'entre eux (Antifer, Cherbourg et Flamanville) et de l'Atlantique pour les 6 autres (Le Conquet, Esquibien, Saint-Guénolé, Saint-Gilles-Croix-de-Vie, Bayonne et Saint-Jean-de-Luz). Il faut souligner que les sites présentant les niveaux de puissance de houle les plus forts (de 20 à $25 \mathrm{~kW} / \mathrm{m}$ sur les sites), se situent à la pointe Bretagne et dans le Pays Basque.

L’énergie transférée du large à la côte est fortement atténuée pour les sites bretons, passant de 44,3 kW/m au large à 21,1 kW/m sur le site de Saint-Guénolé et à 6,9 kW/m sur le site d'Esquibien. Par contre, l'énergie transférée est conservée pour les sites basques, passant de 25,8 kW/m au large à 24,3 kW/m sur le site de Bayonne et à 21,8 $\mathrm{kW} / \mathrm{m}$ sur le site de Saint-Jean-de-Luz.

Ces deux types de transfert s'expliquent par les configurations bathymétriques très différentes des sites, avec en Bretagne, le plateau continental et la configuration complexe et découpée des côtes qui atténuent fortement l'énergie des houles du large, et dans le Pays Basque, la présence du gouf du Cap breton dans les Landes qui favorise au contraire leur transfert.

\section{Discussion}

\subsection{Limites de validité de la méthodologie}

a) Formulation simplifiée

La méthode employée de propagation de la houle du large à la côte (GODA, 2000) présente certaines limites dans la représentation des processus de réfraction, de convergence et divergence de houle et de transfert d'énergie. Les formules de calculs sont limitées pour mettre en évidence les zones de concentration d'énergie de houle autour des caps ou digues et de dissipation d'énergie pénétrant dans les baies.

La formule de calcul du niveau de puissance de houle, employée pour caractériser les sites, reste une formule simplifiée approximative, issue de l'approche spectrale, mais qui reprend la vitesse de groupe définie pour la houle régulière. L'utilisation de la formulation spectrale du niveau de puissance aurait été plus correcte, mais elle requiert l'utilisation de modèles numériques spectraux complexes et longs à mettre en œuvre (EPRI, 2011).

La diffraction autour des obstacles, qui peut s’interpréter comme un processus de transfert d'énergie des zones les plus agitées vers les moins agitées, reste compliquée à formuler et n'est donc pas intégrée à la méthode, en raison de la difficulté de prendre en compte les résultats d’abaques dans les calculs. 
b) Sites de configuration complexe

Les résultats peuvent être surestimés pour des sites de configuration complexe, en raison de la présence d’obstacles ou de hauts fonds qui ne sont pas intégrés dans la méthode de calcul de propagation de houle ; ces obstacles peuvent atténuer et amortir considérablement les houles du large aux abords des sites comme Le Conquet, Cherbourg ou Roscoff, voire au contraire sous-estimer les résultats aux abords de sites comme Esquibien protégé par le cap de Lervily.

\subsection{Perspectives}

Les codes de calcul numérique de propagation de houle et/ou d'agitation les plus appropriés, comme TOMAWAC, REFONDE, SWASH ou WAVEWATCH III, pourront être utilisés pour réaliser les calculs de transfert de potentiel.

La mise en place de bouées directionnelles et de réseaux de capteurs de pression sur les sites pilotes d'Esquibien, Saint-Guénolé et Saint-Jean-de-luz, sera nécessaire pour calibrer les modèles et comparer les résultats.

L’évaluation des résultats de la base de données HOMERE développé par l'IFREMER dans le cadre du projet PREVIMER (http://www.previmer.org/), sera recherchée pour conforter les résultats obtenus, en s'inspirant de la démarche d'étude de l'Electric Power Research Institute (EPRI, 2011). L'analyse de ces données de houle reconstituées sur la période de 1994 à 2012 au plus proche des digues considérées, permettra d’affiner les résultats de potentiels énergétiques des 9 sites sélectionnés.

\section{Références bibliographiques}

BENOIT M., LAFON F., GOASGEN G. (2008). Constitution et exploitation d'une base de données d'états de mer le long des côtes françaises par simulation numérique sur 23 ans. Base ANEMOC en Atlantique, Manche et Mer du Nord. European Journal of Environmental and Civil Engineering, Vol. 12/1-2, pp 35-50. http://dx.doi.org/10.1080/19648189.2008.9692994

EQUIMAR (2011). Deliverable D2.2: Wave and Tidal Resource Characterisation, 83 p. EPRI (2011). Technical Report Mapping and assessment of the United States Ocean Wave Energy Resource, Palo Alto, CA: 2011.1024637. 176 p.

GODA Y. (2000). Random seas and design of maritime structures, Advanced series on Ocean Engineering, Vol. 15, World Scientific, Singapore, 443 p. http://dx.doi.org/10.1142/3587

LENEE-BLUHM P., PAASCH R., TUBA ÖZKAN-HALLER H. (2011). Characterizing the wave energy resource of the US Pacific Northwest, Renewable Energy, Volume 36, pp 2106-2119. http://dx.doi.org/10.1016/j.renene.2011.01.016

MATTAROLO G., BENOIT M., LAFON F. (2009). Wave energy resource off the French coasts: the ANEMOC database applied to the energy yield evaluation of Wave Energy Converters, 8th European Wave and Tidal Energy Conference Series, Uppsala, Sweden. 